\title{
Supervised machine learning classification algorithms for detection of fracture location in dissimilar friction stir welded joints
}

\author{
Akshansh Mishra \\ Centre for Artificial Intelligent Manufacturing Systems, Stir Research Technologies, India \\ akshansh@stirresearchtech.in, https://orcid.org/0000-0003-4939-359X \\ Apoorv Vats \\ Department of Computer Science and Engineering, Jaypee University of Information Technology, India \\ apoorvvats181@gmail.com, bttps:/ /orcid.org/0000-0003-4339-4110
}

\begin{abstract}
Machine Learning focuses on the study of algorithms that are mathematical or statistical in nature in order to extract the required information pattern from the available data. Supervised Machine Learning algorithms are further sub-divided into two types i.e. regression algorithms and classification algorithms. In the present study, four supervised machine learning-based classification models i.e. Decision Trees algorithm, K- Nearest Neighbors (KNN) algorithm, Support Vector Machines (SVM) algorithm, and Ada Boost algorithm were subjected to the given dataset for the determination of fracture location in dissimilar Friction Stir Welded AA6061T651 and AA7075-T651 alloy. In the given dataset, rotational speed (RPM), welding speed $(\mathrm{mm} / \mathrm{min})$, pin profile, and axial force $(\mathrm{kN})$ were the input parameters while Fracture location is the output parameter. The obtained results showed that the Support Vector Machine (SVM) algorithm classified the fracture location with a good accuracy score of 0.889 in comparison to the other algorithms.
\end{abstract}

KEYwORDS. Fracture Location; Machine Learning; Classification; Friction Stir Welding; Dissimilar Joints; Python Programming

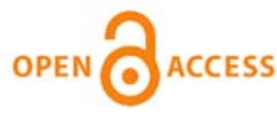

Citation: Mishra, A., Vats, A., Supervised machine learning classification algorithms for detection of fracture location in dissimilar friction stir welded joints, Frattura ed Integrità Strutturale, 58 (2021) 242-253.

Received: 09.07.2021

Accepted: 23.08 .2021

Published: 01.10.2021

Copyright: (C) 2021 This is an open access article under the terms of the CC-BY 4.0, which permits unrestricted use, distribution, and reproduction in any medium, provided the original author and source are credited.

\section{INTRODUCTION}

achine Learning algorithms are vital tools or methods for understanding and handling the data. It should be noted that a statistic can be a graphical or numerical summary of a collection of the given data [1-3]. Data is overwhelming in nature which involves summarization and reduction which makes the dataset comprehensible to a human observer. Understanding the data is very important because sometimes the available data can be misleading. So, the implementation of Machine Learning algorithms provides statistical framework for assessing the quality of available data [4-5]. 
Data is further classified into two key types i.e. Organic/Processed data and "Designed" data collection [6]. Organic data is extracted by a computerized system or from audio and video recordings. This type of data is generated organically as a result of some process and is generated over time. These processes generate massive quantities of data which is called Big Data. In order to process this type of data, significant computational resources are required. On the other hand, "designed" data collection is used to specifically address a stated research objective for example a particular individual sampled from the population.

Machine Learning algorithms have gained some popularity in the Friction Stir Welding Process [7-9]. Machine Learning classification-based approach was used for predicting the mechanical properties of friction stir welded copper joints [10]. The results showed that the Artificial Neural Network model resulted in a good accuracy score of 0.94. Balachandar et al. [11] monitored the Friction Stir Welding tool condition by using the Random Forest algorithm which gave better results. There is limited number of research studies available on the application of Supervised Machine Learning Classification models in the Friction Stir Welding Process. Du et al. [12] used machine learning based classification models for studying the conditions of void formation in Friction Stir Welding process. The results showed that the implemented Artificial Neural Network (ANN) and Decision Tree (DT) algorithms resulted in the accuracy of 96.6\%. Machine Learning based classification algorithms were used for the determination of mechanical properties of Friction Stir Welded Copper joints [13]. The results showed that the Artificial Neural Network (ANN) resulted in the accuracy of $96.6 \%$.

In the present work, the fracture position of dissimilar Friction Stir Welded joints is determined by using four Supervised Machine Learning Classification algorithms which will be discussed in the next sections.

\begin{tabular}{|c|c|c|c|c|}
\hline $\begin{array}{c}\text { Rotational Speed } \\
\text { (RPM) }\end{array}$ & $\begin{array}{l}\text { Welding } \\
\text { Speed } \\
(\mathrm{mm} / \mathrm{min})\end{array}$ & $\begin{array}{l}\text { Pin } \\
\text { Profile }\end{array}$ & $\begin{array}{c}\text { Axial } \\
\text { Force } \\
(\mathrm{kN})\end{array}$ & $\begin{array}{l}\text { Fracture } \\
\text { Position }\end{array}$ \\
\hline 800 & 90 & 1 & 12 & 0 \\
\hline 800 & 100 & 1 & 12 & 1 \\
\hline 800 & 110 & 1 & 12 & 1 \\
\hline 800 & 90 & 2 & 12 & 1 \\
\hline 800 & 100 & 2 & 12 & 1 \\
\hline 800 & 110 & 2 & 12 & 1 \\
\hline 800 & 90 & 3 & 12 & 0 \\
\hline 800 & 100 & 3 & 12 & 0 \\
\hline 800 & 110 & 3 & 12 & 0 \\
\hline 900 & 90 & 1 & 12 & 1 \\
\hline 900 & 100 & 1 & 12 & 1 \\
\hline 900 & 110 & 1 & 12 & 1 \\
\hline 900 & 90 & 2 & 12 & 1 \\
\hline 900 & 100 & 2 & 12 & 1 \\
\hline 900 & 110 & 2 & 12 & 1 \\
\hline 900 & 90 & 3 & 12 & 1 \\
\hline 900 & 100 & 3 & 12 & 1 \\
\hline 900 & 110 & 3 & 12 & 1 \\
\hline 1000 & 90 & 1 & 12 & 0 \\
\hline 1000 & 100 & 1 & 12 & 0 \\
\hline 1000 & 110 & 1 & 12 & 0 \\
\hline 1000 & 90 & 2 & 12 & 1 \\
\hline 1000 & 100 & 2 & 12 & 1 \\
\hline 1000 & 110 & 2 & 12 & 1 \\
\hline 1000 & 90 & 3 & 12 & 1 \\
\hline 1000 & 100 & 3 & 12 & 1 \\
\hline 1000 & 110 & 3 & 12 & 0 \\
\hline
\end{tabular}

Table 1: Experimental Dataset. 


\section{EXPERIMENTAL PROCEDURE}

1 he material used for welding purpose belonged to 6xxx and 7xxx aluminium series. In order to prepare the plate specimens machining process was done to remove the uneven surfaces. The experimental dataset is prepared on

27 Friction Stir Welded specimens [12]. For testing purpose the transverse tensile specimens were prepared according to ASTM E8M-04 standard shown in Fig. 1. Tensile testing was carried out at a room temperature according to ASTM D 557 M- 94 standards. In the present research work, Python programming language was executed on Jupyter lab notebook for subjecting the Supervised Machine Learning algorithms on the given dataset [12]. Jupyter is a web-based interactive development environment that supports multiple programming languages used in which commonly used language is Python Programming. Tab. 1 shows the parameters involved in the experimental study. Rotational Speed (RPM), Welding Speed ( $\mathrm{mm} / \mathrm{min})$, Pin profile, and Axial Force (kN) are input parameters while Fracture position is an output parameter.

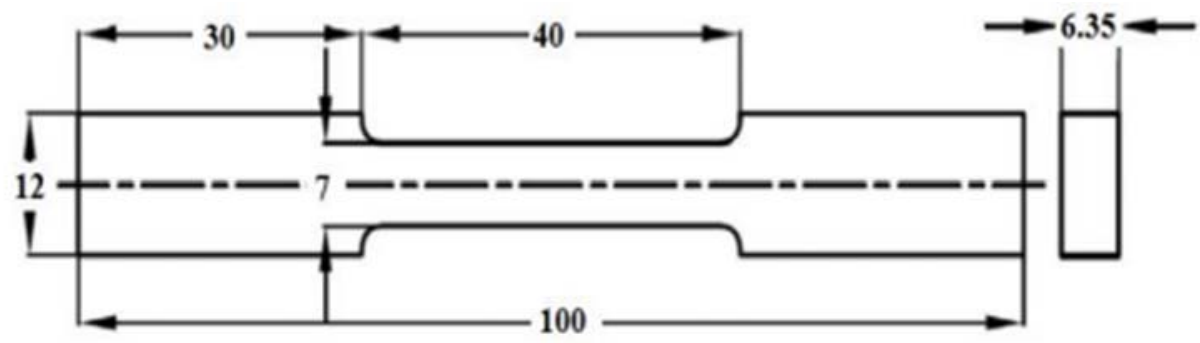

Figure 1: Tensile Test specimen [12]

Pin profiles such as Simple Square is labelled as 1, Taper Cylindrical Threaded is labelled as 2 and Taper Square Threaded is labelled as 3 while the output column i.e. in Fracture position column, the fracture occurring in Stir Zone (SZ) is labelled as 0 and fracture occurring at Heat Affected Zone (HAZ) of 6061 is labelled as 1 . Specimens after and before tensile testing are shown in Fig. 2 a) and 2 b).

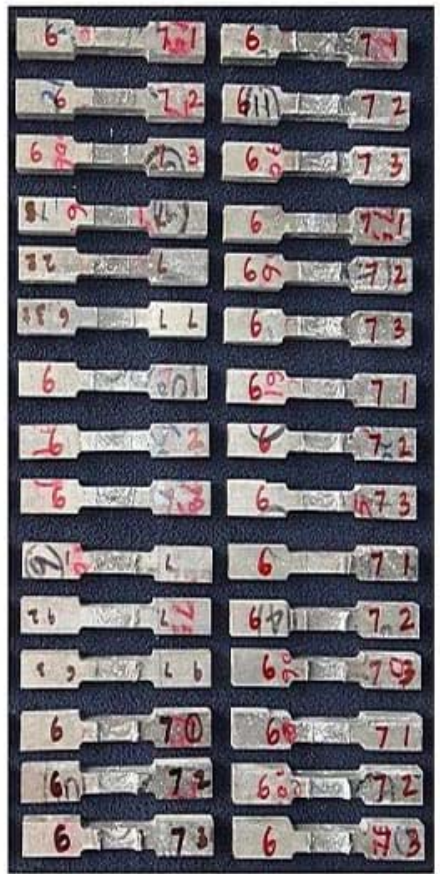

(a)

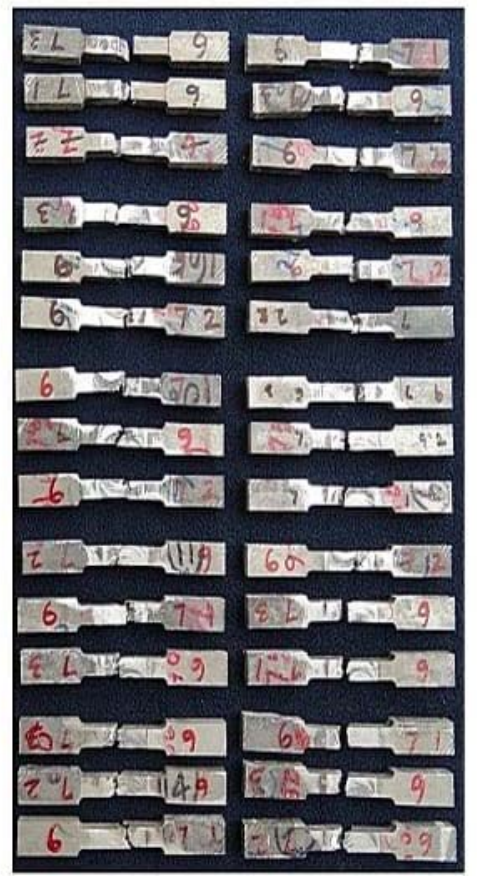

(b)

Figure 2: a) Specimens before testing; b) Specimens after testing.

The required Python libraries such as pandas, NumPy, seaborn, and pyplot were imported. After importing the libraries, the dataset was loaded into the Jupyter environment. Pair plot function is used for establishing the relationship between 
the multiple pairwise bivariate distributions in a given dataset as shown in Fig. 3. Count function was subjected to the given dataset to obtain the number of 0 s and $1 \mathrm{~s}$ in the output column i.e. in the Fracture position column as shown in Fig. 4.
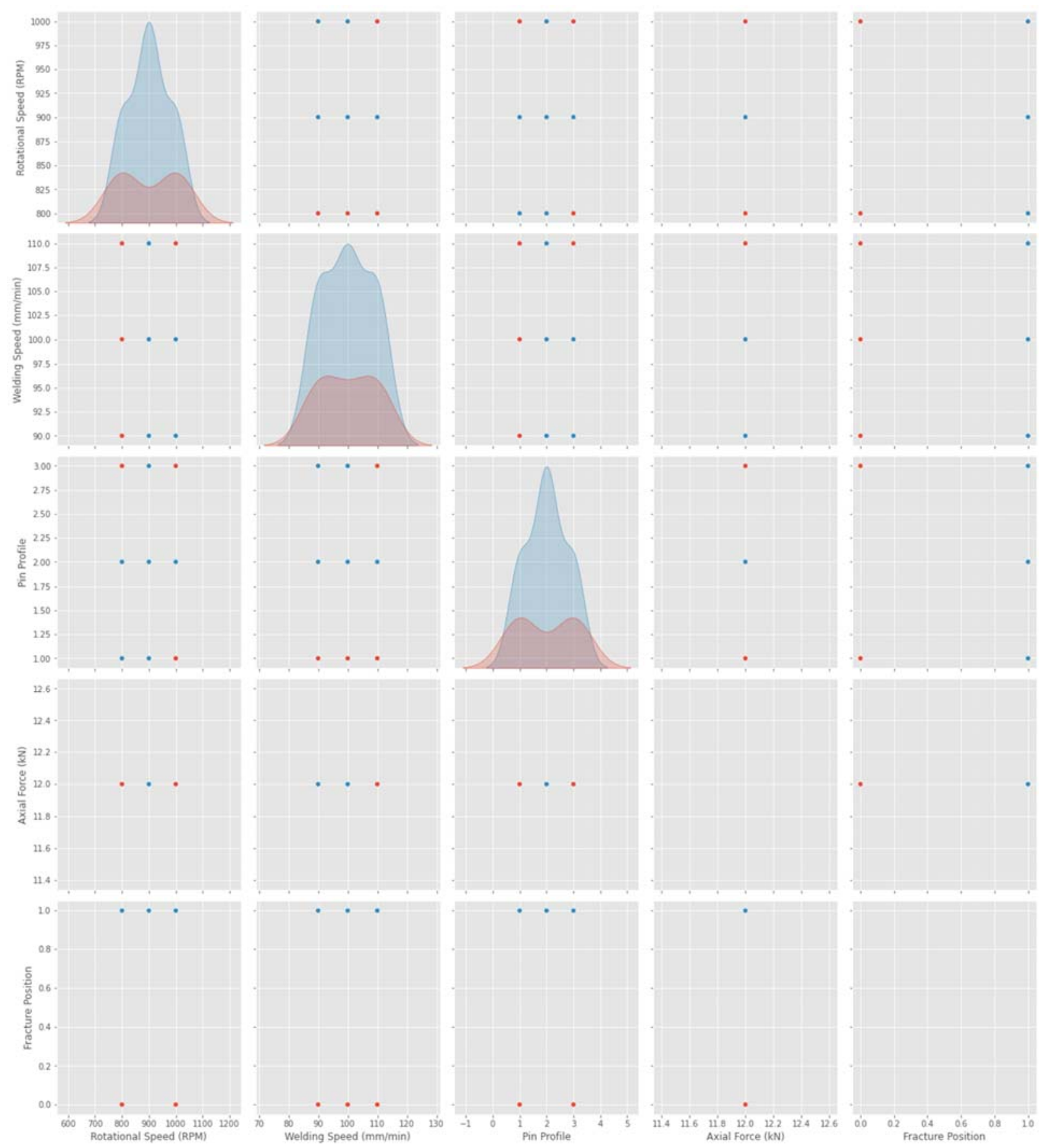

Figure 3: Multiple pairwise bivariate distributions. 


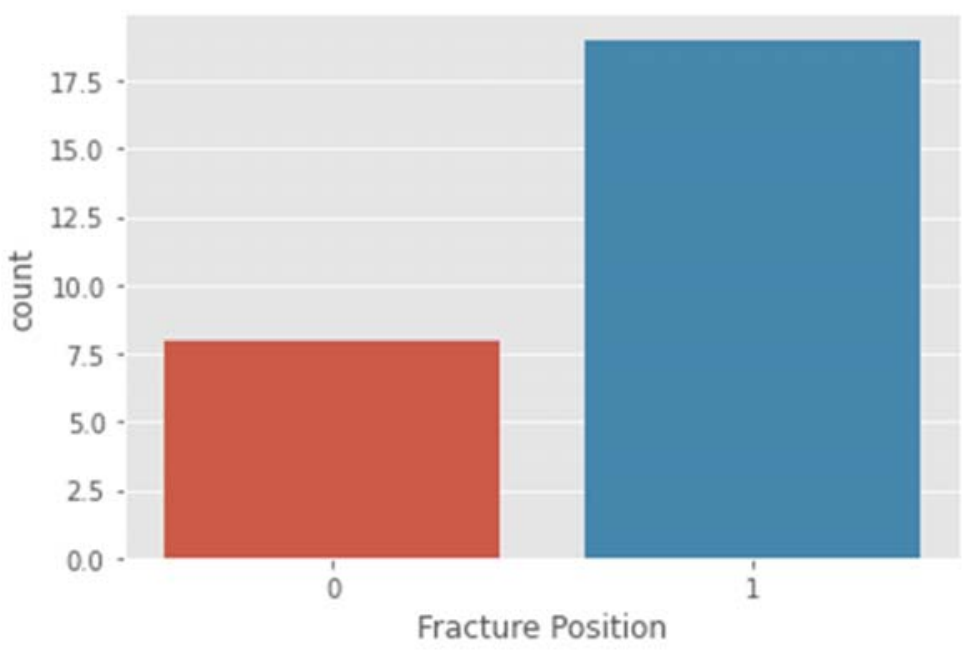

Figure 4: Counting numbers of 0 s and $1 \mathrm{~s}$.

As shown in Fig. 5, Exploratory Data Analysis is used to know about the data pattern and further summarize their main characteristics.
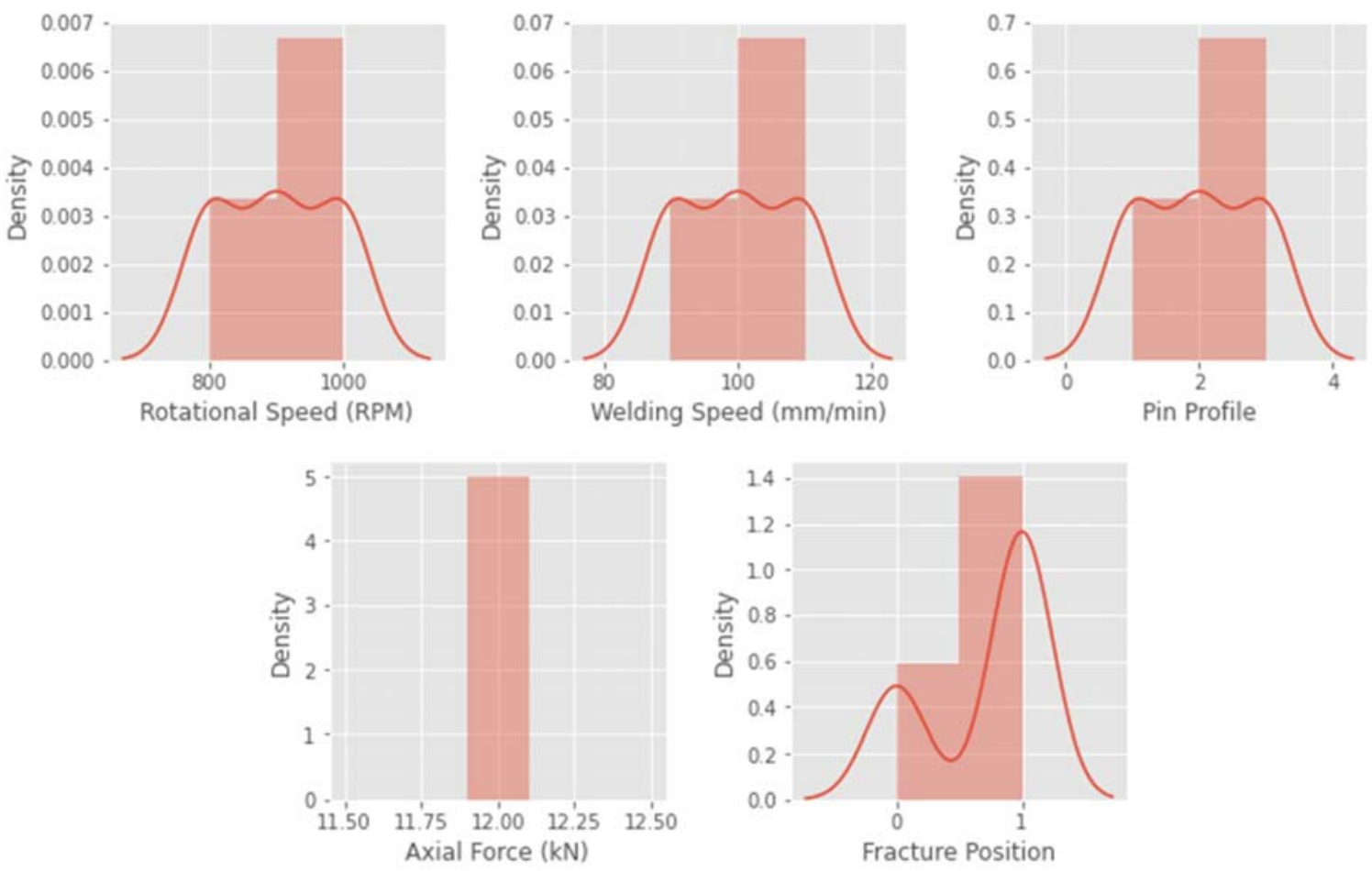

Figure 5: Exploratory Data Analysis

In order to find out the important input parameters affecting the output parameter, the feature importance method was used. From Fig. 6 it is observed that the Axial Force $(\mathrm{kN})$ parameter creates no effect on the output parameter. So due to this reason, this parameter is dropped from the input parameters which are subjected to Machine Learning algorithms. 


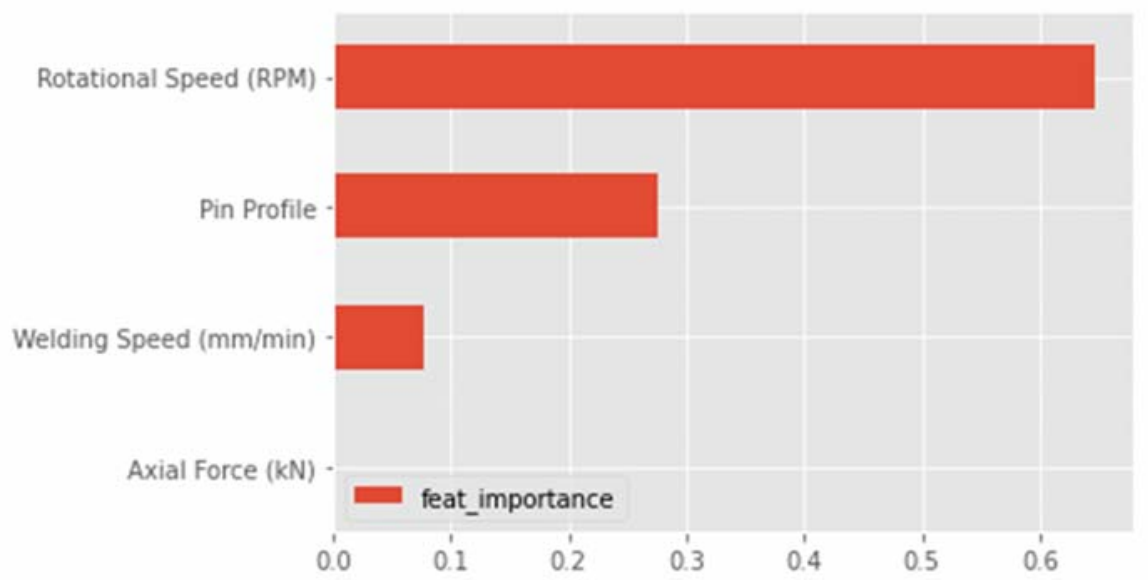

Figure 6: Calculation of Feature Importance

\section{RESULTS AND DISCUSSION}

\section{Decision tree algorithm}

Decision Tree is a supervised machine learning algorithm that can be both implemented for classification and
regression analysis. A Decision Tree model consists of a Terminal node, a decision node, and a root node as seen 1 in Fig. 7.

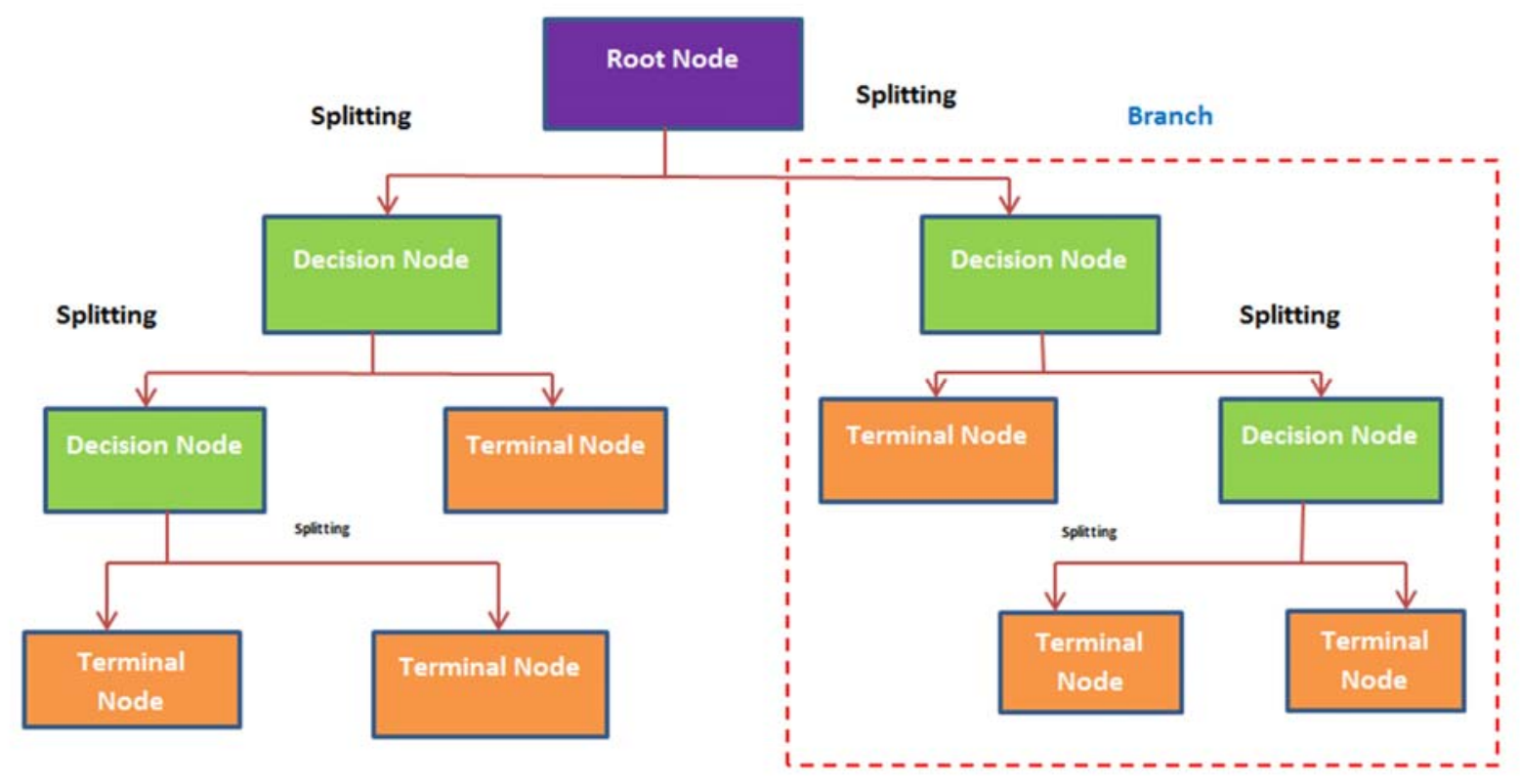

Figure 7: Decision Tree Algorithm architecture.

The main objective is to create many pure nodes with the help of the splitting process. The nodes can be further categorized as pure or homogenous nodes and impure or heterogeneous nodes. After the splitting operation, the pure node contains the data points which belong to the same class while the impure node contains the data points that belong to a different class. The three important parameters which are used to determine the level of impurity are Gini Index, Entropy, and Classification Error. It should be noted that the Information Gain and Entropy are related to each other as seen in Eqn. 1 while entropy can be further calculated by Eqn. 2 . 


$$
\text { Information Gain }=1-\text { Entropy }
$$

$$
\text { Entropy }=-p \cdot \log _{2} p-q \cdot \log _{2} q
$$

where probabilities of success and failure are represented by $\mathrm{p}$ and $\mathrm{q}$ respectively.

Fig. 8 shows the decision tree plot for the given parameters in the dataset. Fig. 9 shows the confusion matrix which is used to describe the performance of the classification model. Tab. 2 shows the classification report of the decision tree algorithm.

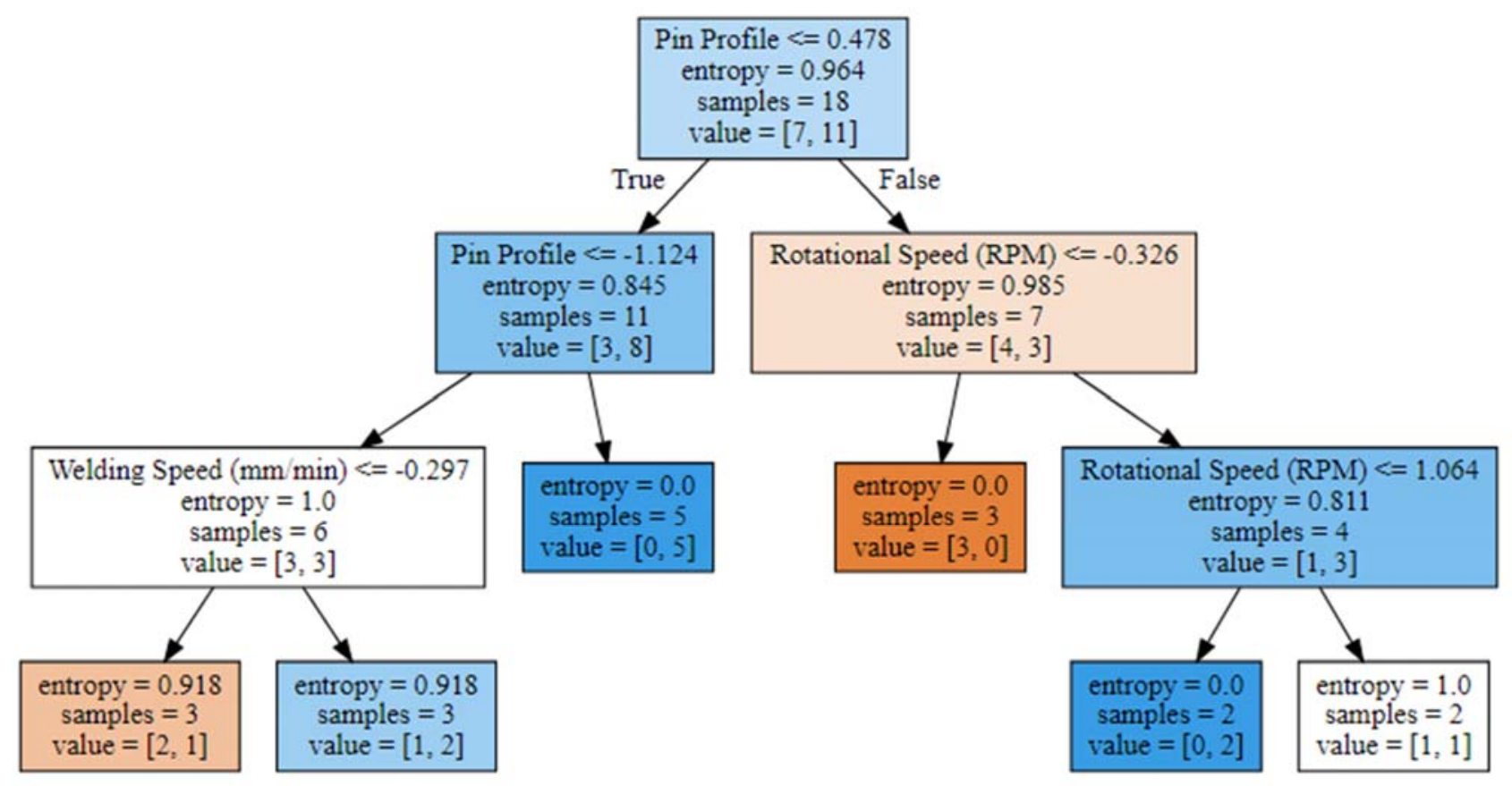

Figure 8: Decision Tree plot for the experimental study.

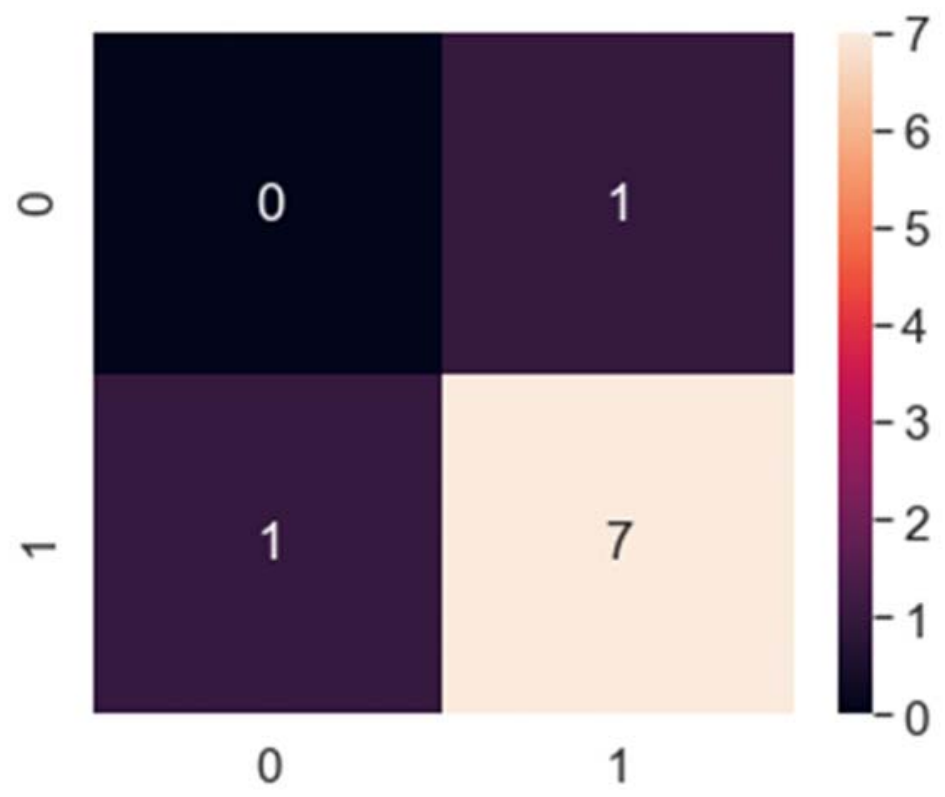

Figure 9: Confusion Matrix showing the performance of Decision Tree algorithm. 


\begin{tabular}{lccc}
\hline & Precision & Recall & F1-score \\
0 & 0.00 & 0.00 & 0.00 \\
1 & 0.88 & 0.88 & 0.88 \\
Accuracy & & & 0.78 \\
Macro average & 0.44 & 0.44 & 0.44 \\
Weighted average & 0.78 & 0.78 & 0.78 \\
\hline
\end{tabular}

Table 2: Classification report of Decision Tree algorithm.

From the Tab. 2 it is observed that the accuracy score obtained from the Decision Tree algorithm is 0.78 .

\section{K-NEAREST NEIGHBOR ALGORITHM}

K

-Nearest Neighbors (KNN) are the most popularly used Machine Learning algorithm where learning is based on the availability of similar data points. KNN represents the model which is nonparametric in nature and its classification mechanism depends on the simple majority votes obtained from the neighbors. The KNN model can be successfully implemented on classification tasks where there is a complex relationship between the target class and attributes. Fig. 10 a) shows the representation of two classes i.e. fracture position at Stir Zone represented by red dots and fracture position at Heat Affected Zone of 6061 by green dots. Fig. 10 b) shows the yellow new data point which has to be classified.

The new data point can fall into the class of fracture location at Stir Zone or to the class of fracture location at Heat Affected Zone of 6061. In order to perfectly classify to which class this new point belongs, the KNN algorithm is used to make classification on the basis of majority votes from the neighborhood data points. To achieve this state, nearest neighbor points are selected on the basis of distance matrices which can be Euclidean distance as shown in Eqn. 3, Manhattan distance as shown in Eqn. 4, and Minkowski distance as shown in Eqn. 5.

$$
\begin{aligned}
& \text { Eucledian Distance between data points }=\sqrt{\left(x_{2}-x_{1}\right)^{2}+\left(y_{2}-y_{1}\right)^{2}} \\
& \text { Manhattan Distance }=\left|x_{1}-x_{2}\right|+\left|y_{1}-y_{2}\right| \\
& \text { Minkowski Distance }=\left(\sum_{i=1}^{n}\left|x_{i}-y_{i}\right|^{p}\right)^{1 / p}
\end{aligned}
$$

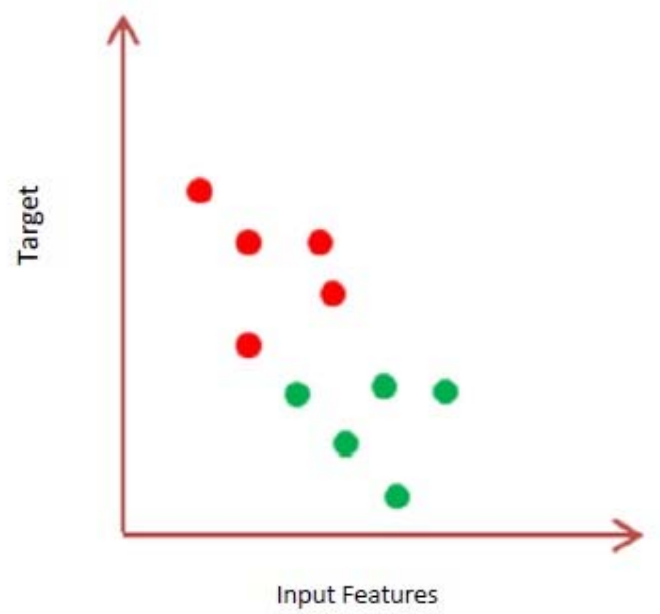

a)

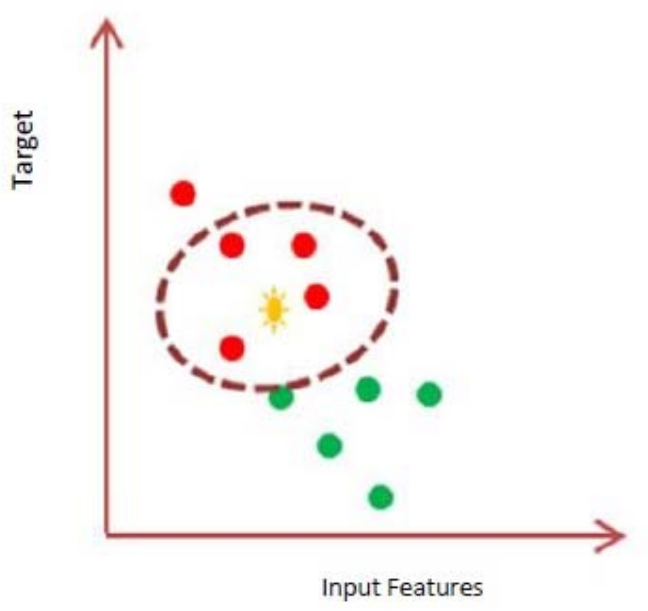

b)

Figure 10: a) Representation of two classes of Fracture Location b) Evaluating the nearest neighbours for a new data point. 
Tab. 3 shows the classification report of K-Nearest Neighbour algorithm and Fig. 11 shows the confusion matrix obtained.

\begin{tabular}{lccc}
\hline & Precision & Recall & F1-score \\
0 & 0.00 & 0.00 & 0.00 \\
1 & 0.86 & 0.75 & 0.80 \\
Accuracy & & & 0.67 \\
Macro average & 0.43 & 0.38 & 0.40 \\
Weighted average & 0.76 & 0.67 & 0.71 \\
\hline
\end{tabular}

Table 3: Classification report of K-Nearest algorithm.

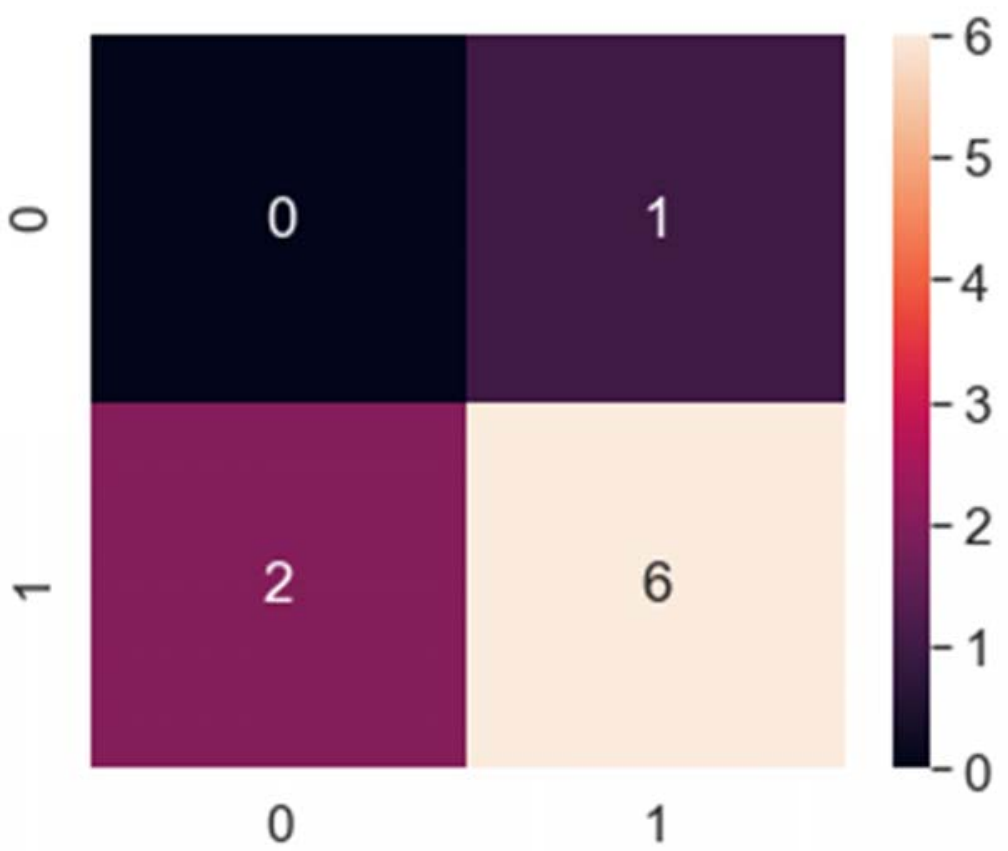

Figure 11: Confusion Matrix showing the performance of K-Nearest Neighbor Algorithm.

From Tab. 3 it is observed that the accuracy score of K-Nearest Neighbor is 0.67 which is less than the accuracy score of Decision Tree algorithm.

\section{SUPPORT VECTOR MACHINE (SVM) ALGORITHM}

$\mathrm{D}$ ecision boundaries generally classify the class based on which the data point falls on as shown in Fig. 12 a) which represents a bad decision boundary as it is unable to properly separate the two classes. There is an infinite number of decision boundaries so the main goal is to choose the best decision boundary. In order to find the optimal line, the Support Vector Machine (SVM) algorithm is used that can perfectly separate the two classes i.e. fracture location at stir zone and fracture position at the heat-affected zone of 6061 as shown in Fig. 12 b).

The decision boundary line can be represented by Eqn. 6 . In order to calculate the distance from the decision boundary to any point Eqn. 7 is used. The main goal is to maximize the value of $r$ for the support vectors points to the required optimal decision boundary.

$$
g(x)=w^{T} x+b=0
$$




$$
r=\frac{g(x)}{\| p \nu \mid}
$$

Tab. 4 shows the classification report of SVM algorithm. From the results it is observed that it resulted in the accuracy score of 0.89. Fig. 13 shows the confusion matrix obtained for the SVM algorithm

\begin{tabular}{l|ccc}
\hline & Precision & Recall & F1-score \\
0 & 0.00 & 0.00 & 0.00 \\
1 & 0.89 & 1.00 & 0.94 \\
Accuracy & & & 0.89 \\
Macro average & 0.44 & 0.50 & 0.47 \\
Weighted average & 0.79 & 0.89 & 0.84 \\
\hline
\end{tabular}

Table 4: Classification report of SVM algorithm.

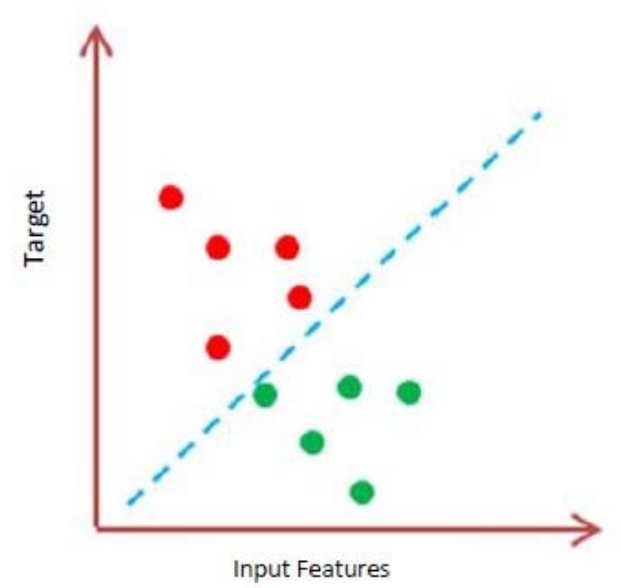

(a)

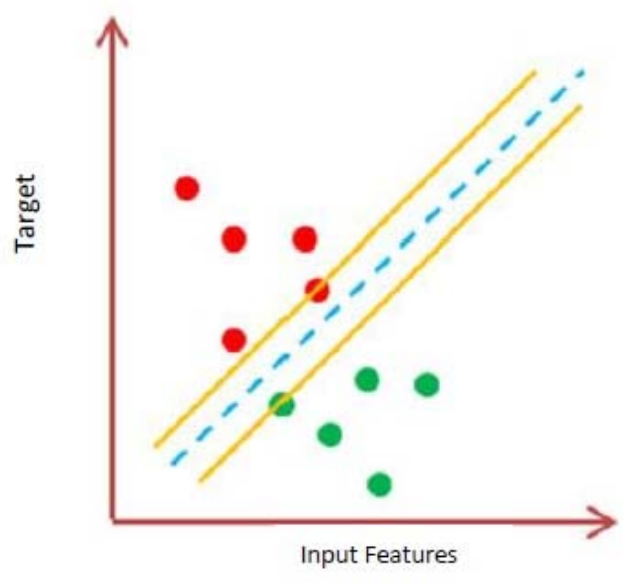

(b)

Figure 12: a) Decision boundary separating two classes of fracture location b) Optimal decision boundary separating the two classes of fracture location.

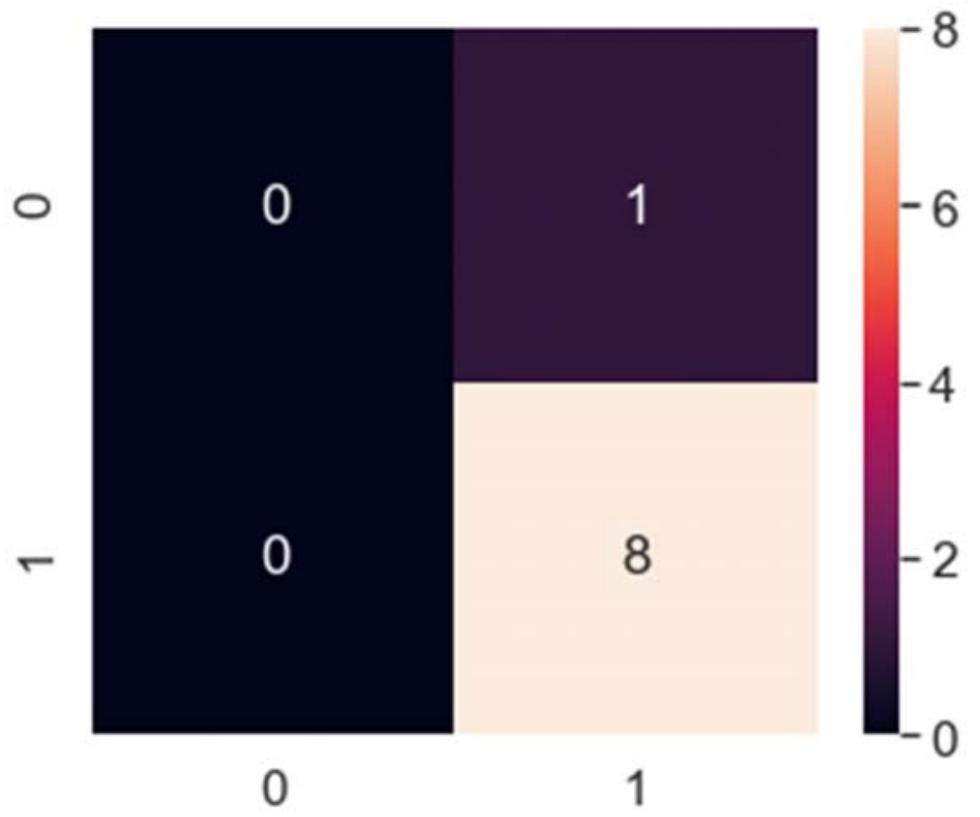

Figure 13: Confusion Matrix showing the performance of SVM algorithm. 


\section{ADA BOOST ALGORITHM}

A

daBoost is also known as Adaptive boosting falls into the classification category of ensemble boosting as shown in Fig. 14. The multiple weak classifiers are combined to increase the accuracy of the classifiers. Adaptive boosting works on an iterative ensemble method that allocates the weights of the classifiers and further data samples are trained in each iteration step.

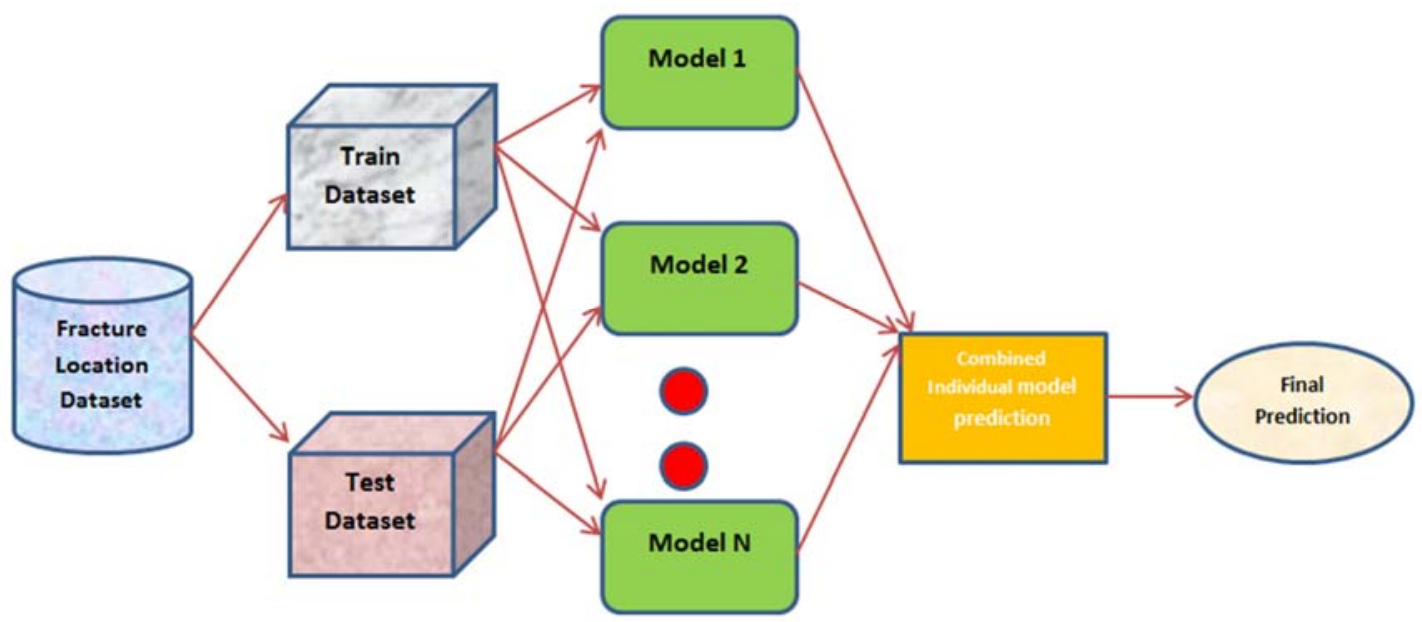

Figure 14: Working Mechanism of Adaptive Boosting algorithm.

Tab. 5 shows the classification report of Ada Boost algorithm and Fig. 15 shows its performance. It is observed that the Ada Boost algorithm resulted in lowest accuracy score of 0.56 in comparison to the other algorithms.

\begin{tabular}{l|ccc}
\hline & Precision & Recall & F1-score \\
0 & 0.00 & 0.00 & 0.00 \\
1 & 0.83 & 0.62 & 0.71 \\
Accuracy & & & 0.56 \\
Macro average & 0.42 & 0.31 & 0.36 \\
Weighted average & 0.74 & 0.56 & 0.63 \\
\hline
\end{tabular}

Table 5: Classification report of Ada Boost algorithm.

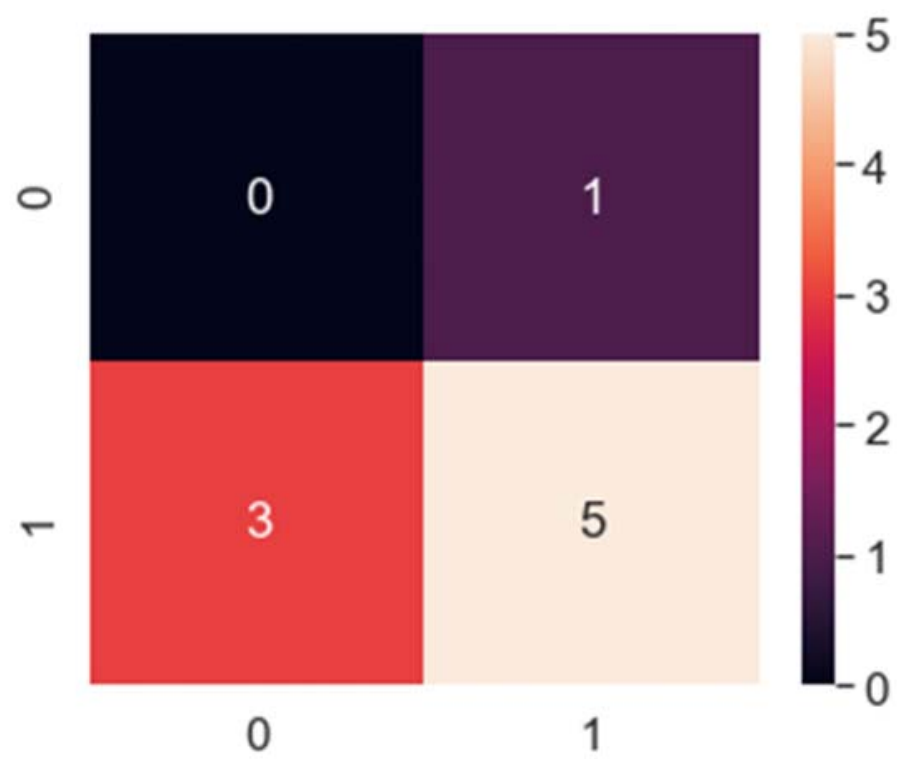

Figure 15: Confusion Matrix showing the performance of Ada Boost Algorithm. 


\section{CONCLUSION}

he recent work successfully implemented the Supervised Machine Learning based classification models for first time detecting the fracture location dissimilar Friction Stir Welded joints.

- The present study successfully implemented the four supervised machine learning classification-based algorithms for the determination of fracture location in dissimilar Friction Stir Welded joints. Support Vector Machine algorithm resulted in highest accuracy score of 0.89 in comparison to the other algorithms while Ada Boost algorithm resulted in the lowest accuracy score of 0.56 .

- It can be concluded that the Support Vector Machine algorithm works really well with a clear margin of separation and is effective in cases where the number of dimensions is greater than the number of samples i.e. in high-dimensional spaces.

- The main insight of the present work is that the Machine Learning based algorithms can reduce the experimental time and cost by resulting in greater accuracy for prediction purpose.

- The future scope of this work is to implement Genetic Algorithms and to check whether they can classify the given task better than the Machine Learning algorithms.

\section{REFERENCES}

[1] Mitchell, T.M. (1999). Machine learning and data mining. Communications of the ACM, 42(11), pp.30-36.

[2] Sajda, P., Gerson, A., Muller, K.R., Blankertz, B. and Parra, L. (2003). A data analysis competition to evaluate machine learning algorithms for use in brain-computer interfaces. IEEE Transactions on neural systems and rehabilitation engineering, 11(2), pp.184-185.

[3] Džeroski, S., Grbović, J., Walley, W.J. and Kompare, B. (1997). Using machine learning techniques in the construction of models. II. Data analysis with rule induction. Ecological Modelling, 95(1), pp. 95-111.

[4] Ratner, B. (2017). Statistical and machine-learning data mining: Techniques for better predictive modeling and analysis of big data. CRC Press.

[5] Gilardi, N. and Bengio, S. (2000). Local machine learning models for spatial data analysis. Journal of Geographic Information and Decision Analysis, 4, pp.11-28.

[6] Xie, J., Song, Z., Li, Y., Zhang, Y., Yu, H., Zhan, J., Ma, Z., Qiao, Y., Zhang, J. and Guo, J. (2018). A survey on machine learning-based mobile big data analysis: Challenges and applications. Wireless Communications and Mobile Computing.

[7] Eren, B., Guvenc, M.A. and Mistikoglu, S. (2021). Artificial intelligence applications for friction stir welding: A review. Metals and Materials International, 27(2), pp.193-219.

[8] Mahadevan, R., Jagan, A., Pavithran, L., Shrivastava, A. and Selvaraj, S.K. (2021). Intelligent welding by using machine learning techniques. Materials Today: Proceedings.

[9] Hartl, R., Bachmann, A., Habedank, J.B., Semm, T. and Zaeh, M.F. (2021). Process Monitoring in Friction Stir Welding Using Convolutional Neural Networks. Metals, 11(4), p.535.

[10] Thapliyal, S. and Mishra, A. (2021). Machine learning classification-based approach for mechanical properties of friction stir welding of copper. Manufacturing Letters.

[11] Balachandar, K. and Jegadeeshwaran, R. (2021). Friction stir welding tool condition monitoring using vibration signals and Random forest algorithm-A Machine learning approach. Materials Today: Proceedings.

[12] Ravikumar, S., Seshagiri, R.V. (2014). Effect of process parameters on mechanical properties of friction stir welded disiimilar materials between AA6061-T651 and AA7075-T651 alloy. Int J Adv Mech Eng 4, pp. 101-114.

[13] Du, Y., Mukherjee, T. and DebRoy, T. (2019). Conditions for void formation in friction stir welding from machine learning. npj Computational Materials, 5(1), pp.1-8.

[14] Thapliyal, S. and Mishra, A. (2021). Machine learning classification-based approach for mechanical properties of friction stir welding of copper. Manufacturing Letters, 29, pp.52-55. 